\title{
Editor's note: 25th Anniversary Special Issue
}

(C) Springer Science+Business Media New York 2015

The Statistics and Computing journal gratefully acknowledges the contributions for this special issue, celebrating 25 years of publication. In the past 25 years, the journal has published innovative, distinguished research by leading scholars and professionals. Papers have been read by thousands of researchers world-wide, demonstrating the global importance of this field. The Statistics and Computing journal looks forward to many more years of exciting research as the field continues to expand.

The eleven papers in this issue include:

Statistics and computing: the genesis of data science, David J. Hand, Founding Editor

EM for mixtures: Initialization requires special care, JeanPatrick Baudry, Gilles Celeux

Sequential Monte Carlo methods for Bayesian elliptic inverse problems, Alexandros Bkos, Ajay Jasra, Ege A. Muzaffer, Andrew M. Stuart

Bayesian inference via projections, Ricardo Silva, Alfredo Kalaitzis

Computing functions of random variables via reproducing kernel Hilbert space representations, Bernhard Schölkopf, Krikamol Muandet, Kenji Fukumizu, Stefan Harmeling, Jonas Peters
The Poisson transform for unnormalised statistical models, Simon Barthelmé, Nicolas Chopin

Scalable estimation strategies based on stochastic approximations: classical results and new insights, Panos Toulis, Edoardo M. Airoldi

de Finetti Priors using Markov chain Monte Carlo computations, Sergio Bacallado, Persi Diaconis, Susan Holmes

Simulation-efficient shortest probability intervals, Ying Liu, Andrew Gelman, Tian Zheng

Flexible parametric bootstrap for testing homogeneity against clustering and assessing the number of clusters, Christian Hennig, Chien-Ju Lin

Bayesian computation: a summary of the current state, and samples backwards and forwards, Peter J. Green, Krzysztof Łatuszyński, Marcelo Pereyra, Christian P. Robert 